\title{
En bloc resection of non-small cell lung cancer invading the thoracic inlet and intervertebral foramina
}

\author{
Elie Fadel, MD \\ Gilles Missenard, MD \\ Alain Chapelier, MD \\ Sacha Mussot, MD \\ François Leroy-Ladurie, MD \\ Jacques Cerrina, MD \\ Philippe Dartevelle, MD
}

Objective: In patients with non-small cell lung cancer invading the thoracic inlet, the transcervical approach does not permit removal of tumor in the intervertebral foramina. We report a variant that lifts this limitation.

Methods: Through the transcervical approach, resectability was assessed and tumorbearing structures were removed, leaving tumor-free margins. Standard upper lobectomy was performed, leaving the lobe in place. A posterior midline approach was used for multilevel unilateral laminectomy, nerve root division inside the spinal canal, and vertebral body division along the midline. The tumor was removed en bloc with the lung, ribs, and vessels through the posterior incision. Fixation of the spine was performed. Medical charts of patients treated with this technique between October 1994 and April 2001 were reviewed retrospectively.

Results: Seventeen patients (mean age 45 years) were treated. Resection of the upper lobe and T1 root was done in all 17 cases; 3 - and 4-level hemivertebrectomies were done in 13 and 3 cases, respectively; 2-level total vertebral body resection and 2-level hemivertebrectomy were done in 1 case; and resections of the phrenic nerve and subclavian artery were done in 7 and 6 patients, respectively. There were no perioperative deaths or residual neurologic impairments. Postoperative complications were pneumonia $(n=6)$, cerebrospinal fluid leakage $(n=1)$, wound breakdown $(\mathrm{n}=1)$, and bleeding necessitating reoperation $(\mathrm{n}=1)$. The overall 3and 5-year survivals were $39 \%$ and $20 \%$, respectively.

Conclusions: Non-small cell lung cancers invading the thoracic inlet and intervertebral foramina can be removed completely through a combined anterior transcervical and posterior midline approach, with good results.

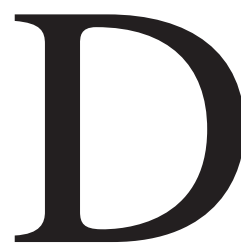

evelopment of the anterior transcervical approach has provided a means of achieving complete resection of non-small cell lung cancer (NSCLC) with spread to the thoracic inlet, producing good results even when the subclavian vessels are invaded. ${ }^{1,2}$ This approach allows en bloc resection of transverse processes or vertebral body segments that adhere to the tumor. Until recently, NSCLC with direct spread to one or more vertebral bodies or intervertebral foramina were considered inoperable. We believe that massive vertebral invasion remains a contraindication to surgery, because it is associated with a short survival time. ${ }^{3}$ However, posteriorly located thoracic inlet tumors with intervertebral foramina invasion along one or more nerve roots but no spread within the spine seemed to us to be amenable to complete en bloc resection. We developed a mixed multidisciplinary approach that combined a posterior midline incision to the anterior 


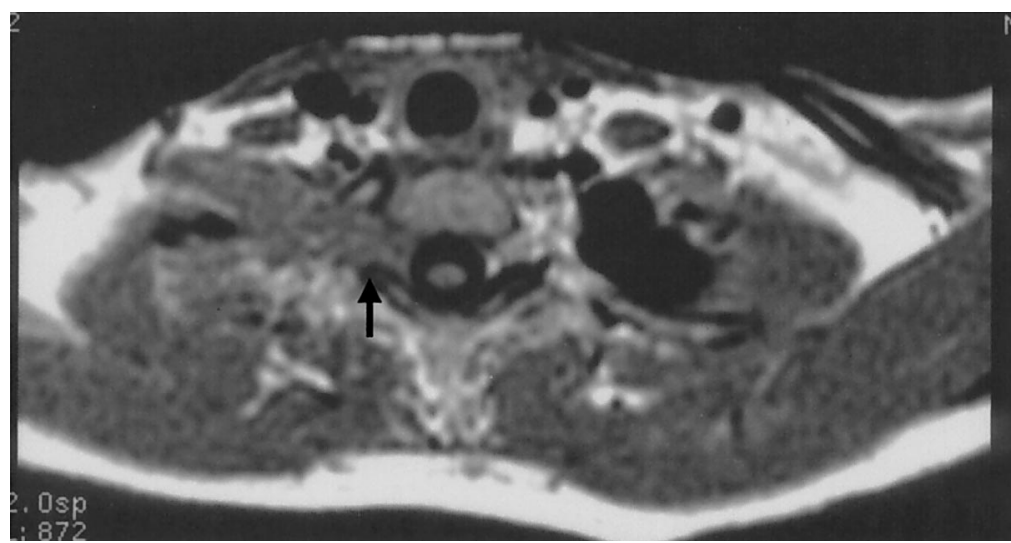

Figure 1. MRI showing NSCLC invading right thoracic inlet and intervertebral foramen along T1 nerve root (arrow).

transcervical approach to allow resection of NSCLCs invading both the thoracic inlet and intervertebral foramina. To minimize the risk of local recurrence, all incisions are made in tumor-free tissue; this requires removal of at least 3 hemivertebrae. Spinal fixation is the last step of the procedure. Our preliminary results are reported and discussed here.

\section{Patients and Methods Patient Data}

Between January 1980 and April 2001, a total of 94 patients with NSCLC invading the thoracic inlet underwent surgery through an L-shaped transcervical approach at our institution. Complete en bloc resection was performed in every case. Five patients with thoracic inlet tumors adherent to the vertebral body underwent transverse process resection with tangential vertebral osteotomy, removing $10 \%$ to $30 \%$ of the vertebral body. These patients were not included in this study because they had no spread to any intervertebral foramina. Seventeen patients (18\%) had direct intervertebral tumor spread to at least 2 intervertebral foramina and were treated since October 1994 with a combined transcervical and posterior midline approach. These 12 men and 5 women, with a median age of 45 years (range 37-59 years), form the basis of this study.

The preoperative workup consisted of chest radiography, bronchoscopy, and head and neck and cervicothoracic computed tomographic (CT) imaging and magnetic resonance imaging (MRI). Vascular invasion was evaluated by venous angiography, aortic arch arteriography, and duplex ultrasonography of the thoracic inlet. Spinal cord arteriography was performed routinely to identify the pattern of spinal cord blood supply. The presence of an anterior spinal artery penetrating into the spinal canal through one of the invaded intervertebral foramina is an established contraindication to surgery. ${ }^{4}$ Intervertebral foramina invasion by the thoracic tumor was diagnosed before the operation in all 17 patients, mainly on the basis of MRI findings (Figure 1). None of the patients had preoperative evidence of vertebral body or spinal canal invasion, which we consider to be a contraindication to surgery. A mediastinoscopy was performed when CT scan showed enlarged mediastinal nodes $(>1.5 \mathrm{~cm})$, and a supraclavicular biopsy was performed when nodes were palpable in the neck.

Eight patients underwent induction chemotherapy. In 1 case radiation therapy was added before referral to our institution. One patient had a history of unsuccessful surgical tumor excision through a posterolateral thoracotomy.

\section{Surgical Technique}

Exposure of the tumor through the transcervical approach was achieved as described previously elsewhere. ${ }^{1-2,5}$ An L-shaped cervicothoracotomy was performed. Division of the sternal attachment of the sternomastoid muscle ensured good exposure of the superior aspect of the subclavian vessels and permitted an assessment of tumor spread and resectability. If the tumor was deemed resectable, the medial half of the clavicle was removed and preserved as a bone autograft. If the subclavian or innominate veins were involved by tumor, they were resected after proximal and distal vessel control; the veins were not reconstructed. The subclavian artery was dissected in a subadventitial plane; when the arterial wall was involved by tumor, however, the subclavian artery had to be resected to ensure excision of the tumor with tumor-free margins. To this end the distal part of the subclavian artery could be exposed after division of the anterior scalene muscle over the first rib. The phrenic nerve was identified and exposed at the anterior aspect of the anterior scalene muscle. Depending on whether it was involved with tumor, it was carefully preserved or resected. The vertebral artery was resected if it was involved or if the adjacent subclavian artery was involved. Arterial reconstruction was preferably performed by direct end-to-end anastomosis at completion of the transcervical approach. This was often possible, because the first 3 ribs were always removed with the tumor. If the segment of artery removed was so long that the two ends were under tension, a polytetrafluoroethylene ringed graft (No. 6 or 8) was used as an interposition graft. En bloc upper lobectomy with mediastinal dissection was performed through the transcervical approach. The hilar, carinal, paratracheal, esophageal, and inferior pulmonary ligament lymph nodes were dissected.

Extension of the tumor to the brachial plexus branches required outside-to-inside dissection, in which secondary branches were 


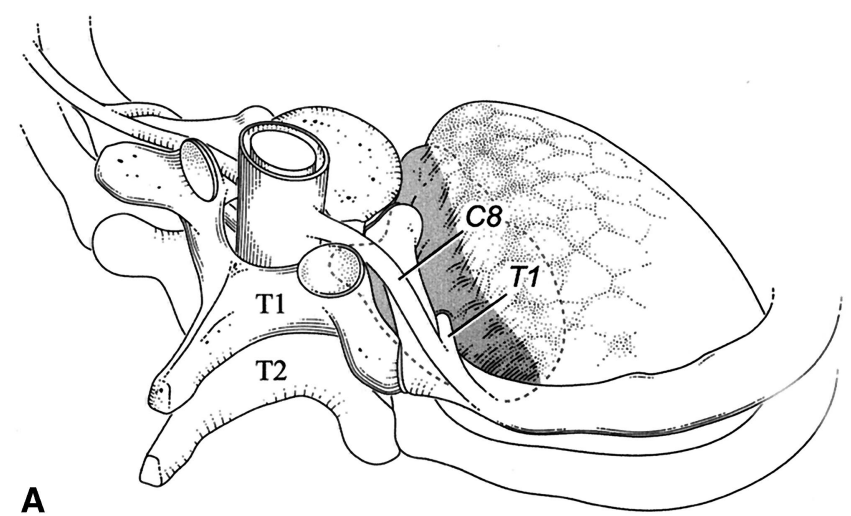

B
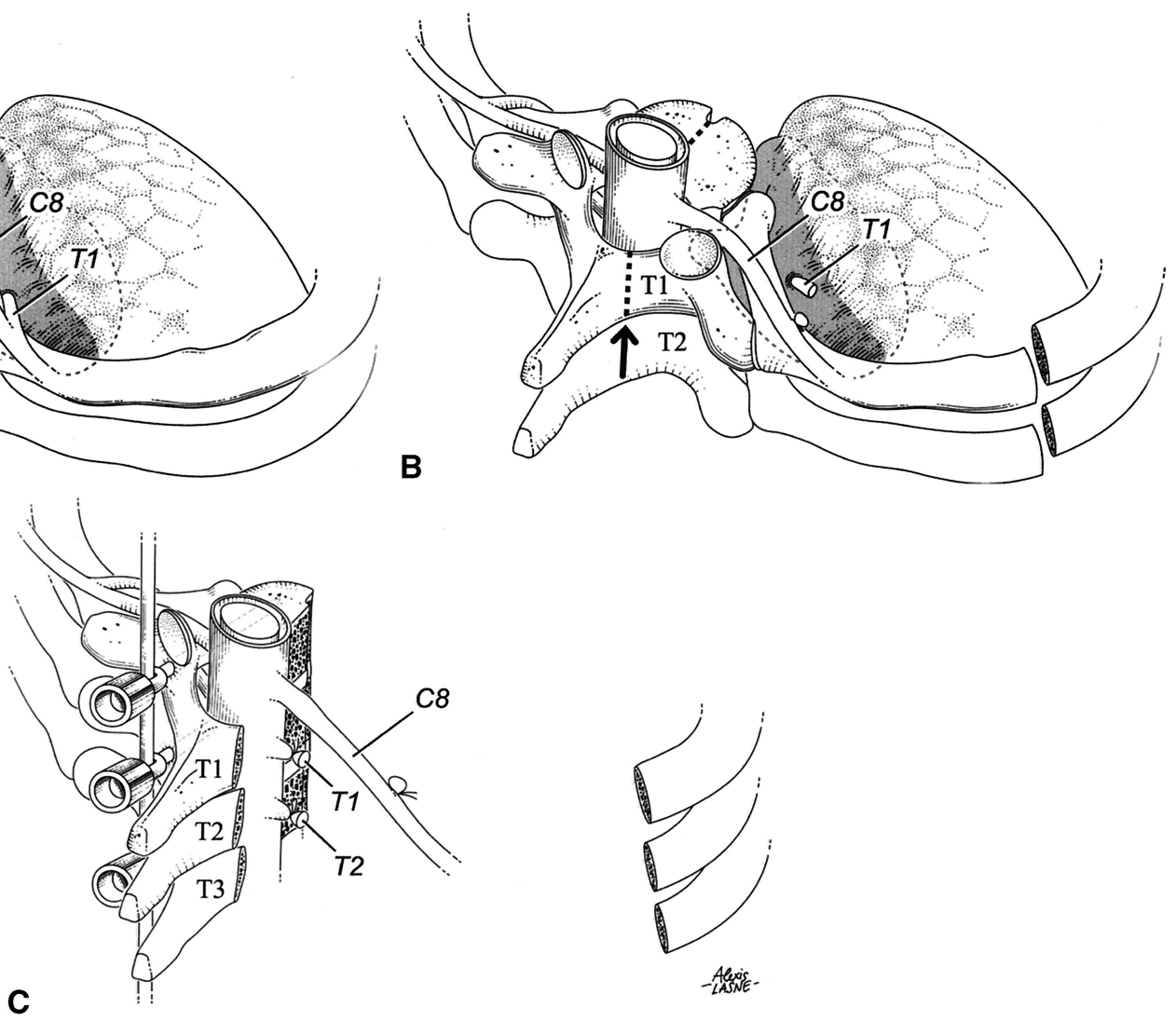

Figure 2. A, Right NSCLC invading thoracic inlet and first two thoracic intervertebral foramina along T1 and T2 nerve roots. B, First step of procedure is anterior transcervical approach that permits assessment of resectability and dissection of tumor in tumor-free margins. Anterior aspect of spine is approached from T1 to T2, and midline groove is made along involved vertebrae to facilitate hemicorporectomy during posterior approach of spine. C, Posterior midline approach is then performed, extending from spinal processes of $\mathrm{C} 7 \mathrm{to} \mathrm{T4}$. After laminectomy of T1 to T3, T1 and T2 nerve roots are divided inside spinal canal. Hemicorporectomy of T1 to T3 and spinal fixation are finally performed.

freed first, followed by primary branches. T1 was always invaded by the tumor and was cut over the first rib (Figure 2). This facilitated dissection of the $\mathrm{C} 8$ root up to the intervertebral foramen. Tumor spread to brachial plexus branches above T1 was considered a contraindication to surgery. Invaded ribs were then resected laterally in tumor-free margins.

Next, the spine was approached medial to the carotid sheath. Care was taken to avoid placing metal retractors along the tracheoesophageal groove. Blunt dissection was performed along the anterior longitudinal ligament of the spine. Anterior dissection along the pretracheal fascia and below the innominate artery allowed the trachea and esophagus (as a unit) to be gently retracted toward the other side. This blunt dissection provided excellent anterior exposure from the C6 vertebra down to the T5 vertebra, if required. The extent of vertebra that had to be removed was evaluated on preoperative MRI scans and confirmed by gentle digital palpation of the intervertebral foramina. A midline slice was made through the prevertebral planes to facilitate section of the involved vertebral bodies (Figure 2). In each of our cases at least 2 intervertebral foramina were invaded by the tumor; conse- quently, at least 3 hemivertebrae had to be removed. At the end of the transcervical approach, the specimen was left in place, and all resected structures still adhered en bloc to the invaded spine. The transcervical approach was then closed.

The patient was placed in the prone position, and a vertical midline incision extending from the spinal process of $\mathrm{C} 7$ to that of T4 was performed. After unilateral multilevel laminectomy extending from the vertebra above to the vertebra below the invaded intervertebral foramina, the nerve roots were exposed and divided inside the spinal canal at their emergence from the external spinal cord sheath, proximal to the dorsal root ganglion. Involved segmental vessels were identified, ligated, and transected. An osteotome was used to cut the vertebral bodies along the midline, working in the posterior-to-anterior direction (Figure 2). The osteotome entered the medial cortex of the involved pedicle and was directed toward locator markers placed anteriorly on the vertebral body. This avoided osteotomy through unsafe areas. The specimen was removed en bloc with the lung, ribs, and vessels through the posterior incision (Figure 3). On the side of the tumor, spinal fixation was performed with hooks in the pedicles above and 


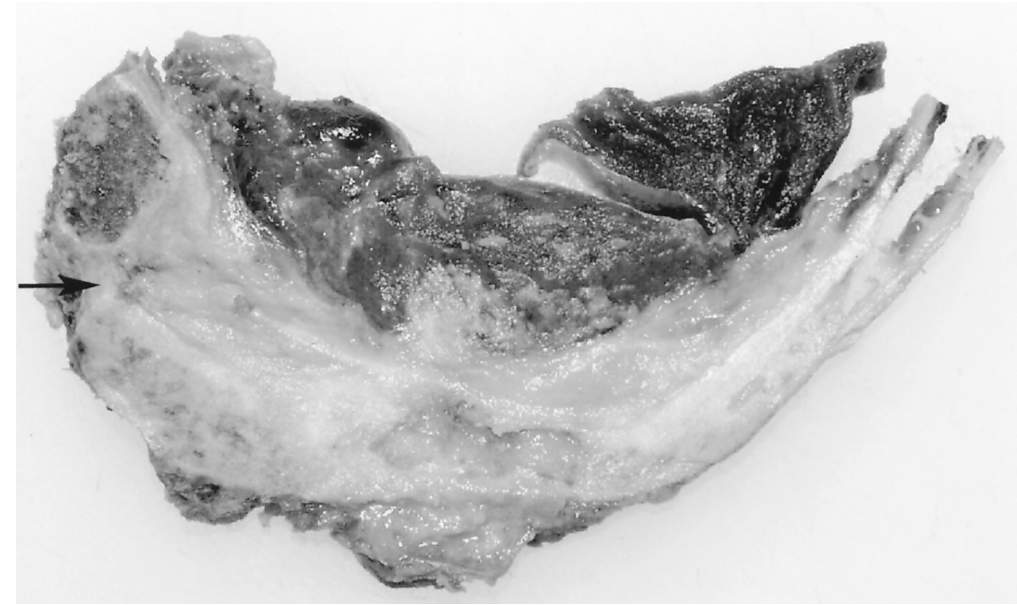

Figure 3. Specimen showing en bloc resected NSCLC invading thoracic inlet and intervertebral foramen (arrow) along thoracic nerve root.

below the hemivertebrectomy area; on the other side, a screw or hook was placed in each pedicle as described by Lapresle and Missenard $^{6}$ and Missenard and colleagues ${ }^{7}$ (Figure 4). Spinal fixation was performed with titanium instrumentation (Diapason; Stryker Spine, Cestas France), which allowed postoperative MRI studies (Figure 5). In all our cases autogenous bone harvested from the iliac spine and from the resected clavicle was used for spinal stabilization. In the case of a patient who required complete vertebral body resection at two levels and hemivertebrectomy at two levels, in addition to iliac bone the clavicle resected during the transcervical phase was interposed between the remaining vertebral bodies to strengthen the spinal fixation.

\section{Statistical Analysis}

All results are expressed as mean \pm SE. Survival was calculated with life-table analysis. Kaplan-Meier curves were plotted. ${ }^{8}$ Follow-up was by routine chest radiography, CT scans, and MRI of the thoracic inlet region.

\section{Results}

Table 1 reports the demographic profile of the study patients. All 17 patients underwent complete en bloc removal of the thoracic inlet tumor and any invaded intervertebral foramina. The tumor was on the right side in 9 patients and on the left in 8 . Hemivertebrectomy was done at 3 levels in 13 patients ( $\mathrm{T} 1$ to $\mathrm{T} 3, \mathrm{n}=12 ; \mathrm{T} 2$ to $\mathrm{T} 4, \mathrm{n}=1$ ) and at 4 levels (T1 to T4) in 3 patients. The remaining patient had a tumor invading the bodies of T2 and T3 and 3 intervertebral foramina. After induction chemotherapy, MRI showed no evidence of vertebral body invasion. Consequently, complete $\mathrm{T} 2$ and $\mathrm{T} 3$ corporectomy was performed, with $\mathrm{T} 1$ and T4 hemivertebrectomy.

The first and second ribs were removed in 2 patients, the first 3 ribs were removed in 6 , and the first 4 ribs were removed in 8 . In 1 patient ribs 2 through 4 were resected. Chest wall reconstruction was not performed. In all patients

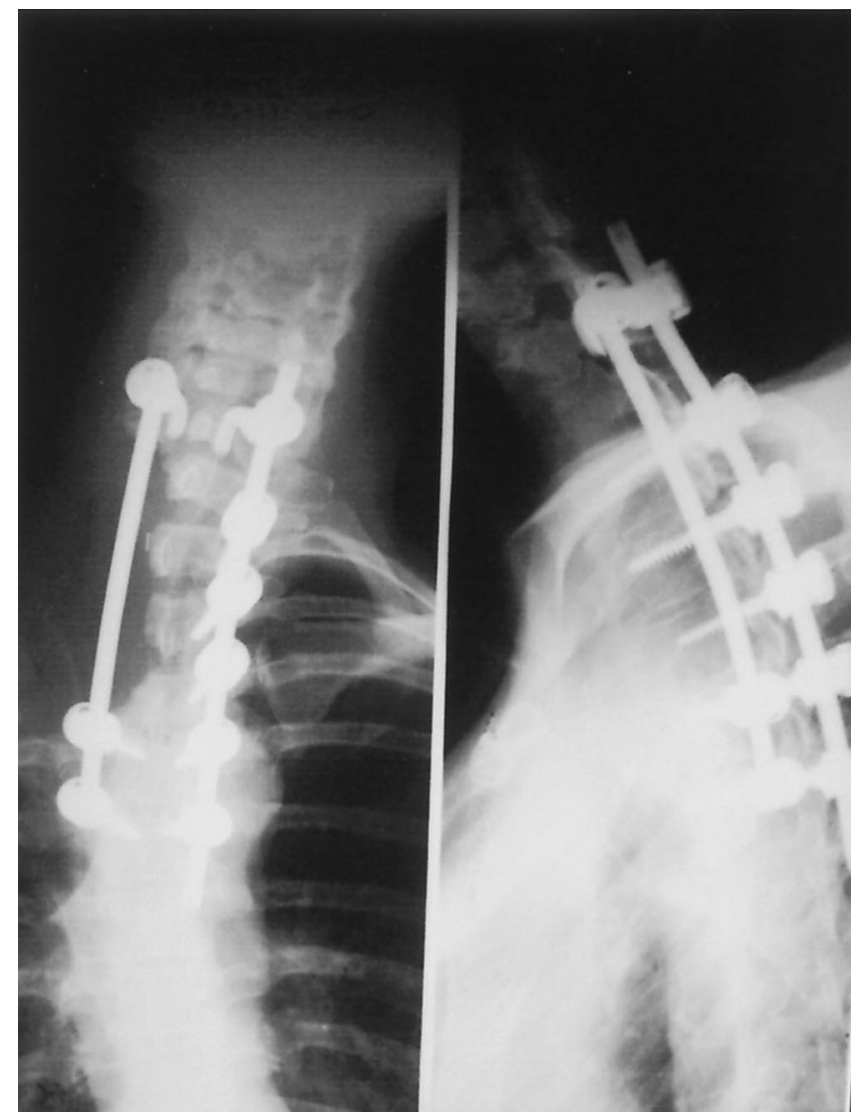

Figure 4. Postoperative chest radiograph showing spinal fixation with hook in pedicle above and below hemivertebrectomy. On other side, screw or hook is implanted in each pedicle.

the lower root (T1) of the brachial plexus was involved by the tumor and was resected; 8 patients required removal of the phrenic nerve as well. In 6 patients the 


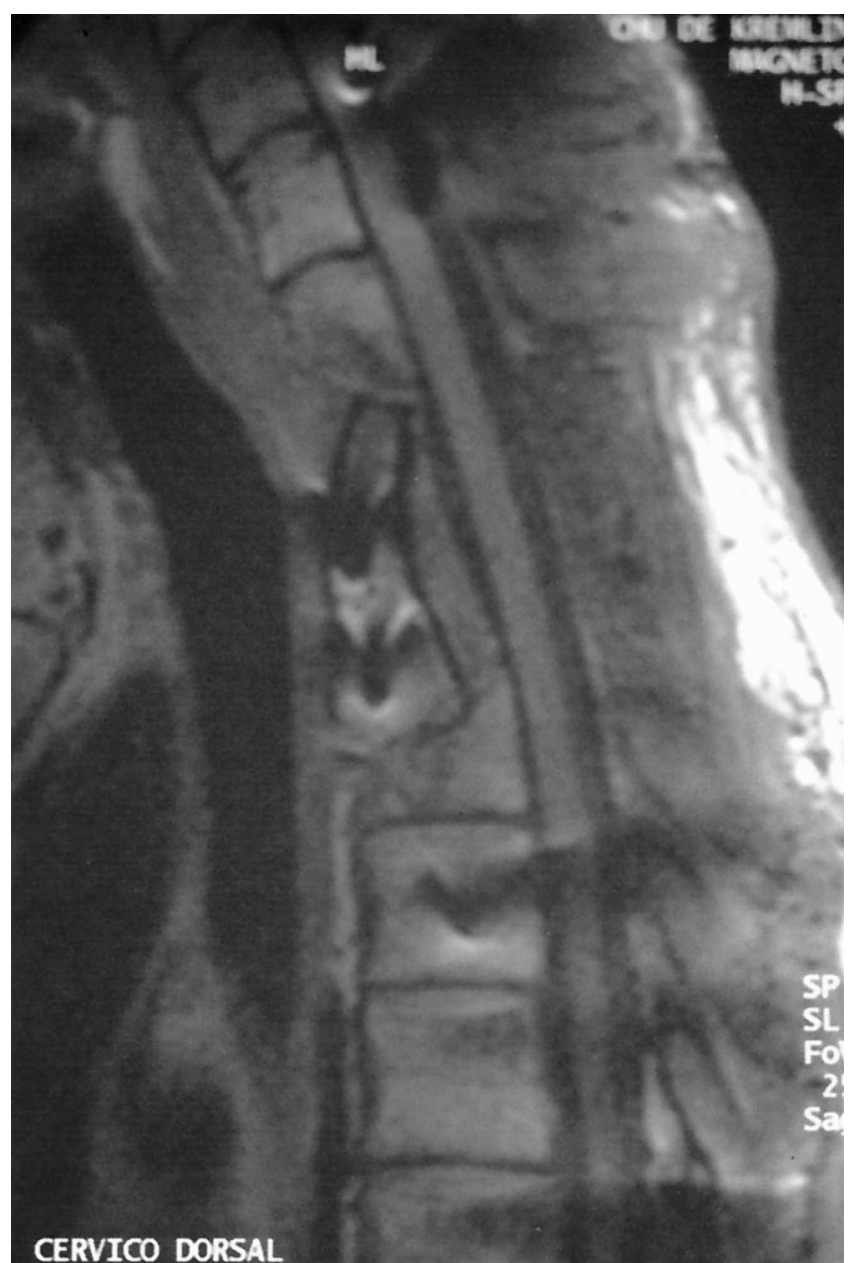

Figure 5. MRI performed 5 years after tumoral resection, showing spinal fixation with hooks, clavicle interposed between remaining vertebral bodies to strengthen spinal fixation, and absence of local recurrence.

subclavian artery was invaded by the tumor. Revascularization was performed with end-to-end anastomosis $(\mathrm{n}=$ $3)$ or a polytetrafluoroethylene graft $(n=3)$ through the transcervical approach. The arterial reconstruction was not shielded from the remaining lung. The subclavian vein and vertebral artery were resected in 3 patients and 2 patients, respectively, and were not reconstructed. Lung resection consisted of lobectomy followed by mediastinal lymphadenectomy in all 17 patients. Two patients had N3 (supraclavicular) disease, 2 had $\mathrm{N} 2$ disease, 1 had N1 disease, and 12 had N0 disease. The median size of the tumor was $5 \mathrm{~cm}$ (range $3-8 \mathrm{~cm}$ ). The spinal canal was found to be free of disease in all patients, confirming the preoperative MRI findings.

Frozen sections of the distal margins of the tumor-bearing areas were examined during the operation. Permanent pathologic sections showed tumor invasion of 2 or more intervertebral foramina in all patients without vertebral body invasion. Nevertheless, permanent sections from 4 patients showed tumor in close contact with and surrounding the vertebral body, without a well-defined margin but also without cortical invasion, even in the patient who had MRI evidence of spread to 2 vertebral bodies before chemotherapy.

There were no perioperative deaths or residual neurologic impairments. Blood loss during the procedure was estimated at 1000 to $15000 \mathrm{~mL}$ (median $4500 \mathrm{~mL}$ ). Operative times ranged from 5 hours and 10 minutes to 12 hours (median 8 hours and 20 minutes). The median length of hospitalization was 30 days (range 14-90 days). Postoperative complications occurred in 9 patients $(53 \%)$ and included pneumonia $(\mathrm{n}=6)$, cerebrospinal fluid leakage $(\mathrm{n}=$ $1)$, wound breakdown $(\mathrm{n}=1)$, and bleeding necessitating reoperation $(n=1)$. Prolonged ventilatory support $(7-30$ days) was necessary for 7 of the 8 patients who underwent phrenic nerve resection. A ventriculoperitoneal shunt was implanted in the patient with cerebrospinal fluid leakage.

Postoperative radiation therapy was used in all 17 patients, with cumulative dosages ranging from 30 to $60 \mathrm{~Gy}$. Four patients each received 5 postoperative chemotherapy cycles.

No patients were unavailable for follow-up. During the study $10(59 \%)$ patients died of tumor recurrence (3 local and distant and 7 distant only). Median survival time was 27 months, and overall 3- and 5-year survivals were 39\% and $20 \%$, respectively (Figure 6). The overall 3- and 5-year disease-free survivals were $30 \%$ and $20 \%$, respectively. Among survivors, 1 patient had a contralateral tumor recurrence after 13 months and underwent tumor resection with postoperative chemotherapy; he was alive and free of recurrence 28 months after the initial mixed-approach procedure. Six patients were alive and free of carcinoma; for 2 of these 6 patients, follow-up was longer than 60 months. Median follow-up was 26 months (range 2-70 months). The overall 3- and 5-year disease-free survivals were $30 \%$ and $20 \%$, respectively. During follow-up, spinal instability developed in 1 patient because of radiation-related osteonecrosis. One year after tumor resection, surgery to realign the spine and change the fixation was successful.

\section{Discussion}

This study shows that the combined anterior transcervical and posterior midline approach is safe and effective for complete en bloc resection of NSCLC invading both the thoracic inlet and at least 2 intervertebral foramina. This aggressive radical procedure produced good results without any operative deaths. Two of these patients with tumors that would classically be considered inoperable ${ }^{3}$ survived for longer than 5 years. The overall 3- and 5-year survivals achieved in this 


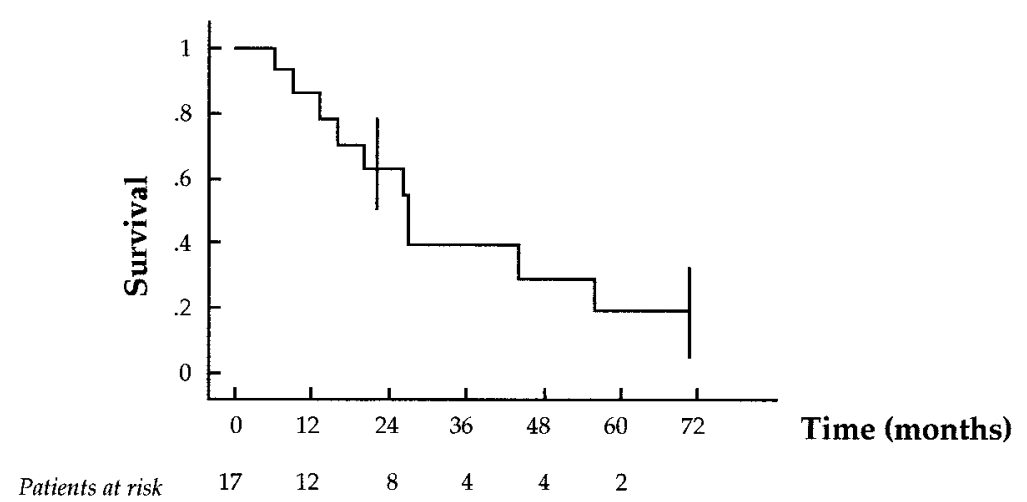

Figure 6. Survival curve for patients with NSCLC invading thoracic inlet and at least 2 intervertebral foramina. Vertical bars indicate SE.

group of patients were similar to those reported among patients surgically treated for NSCLC invading the thoracic inlet but not any intervertebral foramina. ${ }^{9-15}$

Since it was introduced by Dartevelle and colleagues, ${ }^{1}$ the anterior transcervical approach has been found effective and safe for the resection of bronchogenic and nonbronchogenic tumors invading the thoracic inlet. ${ }^{1}$ This approach allows en bloc resection of the thoracic inlet tumor and of involved adjacent structures: the chest wall and T1 nerve root can be resected, the subclavian artery can be resected and reconstructed, ${ }^{2}$ the upper lung lobe can be removed, and the mediastinal lymph nodes can be dissected. Posterolateral thoracotomy was not used in the last 50 patients who underwent surgery in our unit for NSCLC invading the thoracic inlet. This change in practice reflects our gradually increasing experience with the anterior transcervical approach. Furthermore, 5 patients not included in this study were found during the operation to have tumor adhesion to the spine, although preoperative investigations had shown no evidence of bone invasion. The transcervical approach allows transverse process resection and tangential vertebral body osteotomy with complete en bloc resection of thoracic inlet tumors that spare the intervertebral foramina. When the tumor extends posteriorly into the intervertebral foramina along the thoracic nerve roots, however, complete resection cannot be obtained through the transcervical approach. The need to divide the nerve roots inside the spinal canal in a tumor-free area led us to use a posterior approach. Only the combination of an anterior and a posterior approach allows complete en bloc resection of the tumor and invaded intervertebral foramina. We also used this mixed approach successfully to resect a nonbronchogenic cancer invading the thoracic inlet and intervertebral foramina in 4 patients.

Because radiographic evidence of massive bone destruction of the vertebral body is associated with a poor prognosis and a short survival time, ${ }^{3,9,12,13}$ we consider this finding to be an absolute contraindication to surgery. Another ab- solute contraindication is intraspinal extension of the tumor. These contraindications underscore the importance of obtaining a CT scan and coronal and sagittal MRI scans of potentially involved vertebrae. Nevertheless, when the tumor is simply adherent to the vertebral body or transverse process because it invades the parietal pleura or periosteum, the prognosis is more favorable, ${ }^{3}$ and resection can be achieved by tangential osteotomy without spinal fixation, which is less cumbersome than our mixed procedure. Furthermore, when induction chemotherapy is followed by a major decrease in MRI evidence of vertebral body invasion, as in 1 of our patients, tumor resection can be performed as long as both visible tumor and the initially invaded areas are removed. This can be achieved by performing en bloc total vertebral body resection.

The patients in our study did not have spread to the vertebral bone. They had direct tumor spread along the sheath of the nerve roots into the intervertebral foramina, without spinal canal or vertebral body invasion. The nerve root in the spinal foramen was free of tumor. Thus, with our posterior approach and after unilateral laminectomy we were able to cut the nerve root in a tumor-free area, close to the spinal cord dura mater. In contrast to the surgical technique described by Gandhi and coworkers ${ }^{16}$ for resecting invaded vertebrae, our technique allows complete en bloc tumor resection without tumor opening. Gandhi and coworkers ${ }^{16}$ cut through the tumor because they removed the vertebrae separately from the invaded chest wall and lung parenchyma. Grunenwald and colleagues ${ }^{17}$ suggested total vertebrectomy for such tumors. We believe that total vertebrectomy is more aggressive than the multilevel hemivertebrectomy used in our technique; in particular, total vertebrectomy requires the patient to wear a brace for 6 months, ${ }^{17}$ which is not well accepted by these patients because they are aware that their chances of prolonged survival are slim. In addition, total vertebrectomy is not necessary because the vertebral bodies are not invaded; vertebral invasion by NSCLC occurs lo- 
TABLE 1. Demographic profile of the study patients

\begin{tabular}{|c|c|c|c|c|c|c|c|}
\hline Patient & Sex & $\begin{array}{c}\text { Age } \\
\text { (y) }\end{array}$ & Side & $\begin{array}{l}\text { Nodal } \\
\text { status }\end{array}$ & Histologic type & $\begin{array}{l}\text { Tumor size } \\
\text { (cm) }\end{array}$ & Previous treatment \\
\hline 1 & $M$ & 54 & Left & No & Adenocarcinoma & 6 & \\
\hline 2 & $M$ & 55 & Left & N2 & Large cell & 5 & \\
\hline 3 & M & 42 & Left & N2 & Squamous cell & 8 & \\
\hline 4 & $\mathrm{M}$ & 42 & Right & N3 & Mixed cell & 4 & \\
\hline 5 & $\mathrm{M}$ & 42 & Right & NO & Adenocarcinoma & 4 & \\
\hline 6 & $\mathrm{~F}$ & 40 & Right & NO & Squamous cell & 6 & Chemotherapy \\
\hline 7 & $\mathrm{~F}$ & 37 & Right & NO & Adenocarcinoma & 3 & Chemotherapy \\
\hline 8 & $\mathrm{~F}$ & 37 & Left & NO & Adenocarcinoma & 3 & Chemotherapy \\
\hline 9 & $M$ & 40 & Right & NO & Adenocarcinoma & 5 & Chemotherapy \\
\hline 10 & $\mathrm{M}$ & 59 & Left & NO & Adenocarcinoma & 5 & \\
\hline 11 & $\mathrm{M}$ & 55 & Left & NO & Adenocarcinoma & 5 & Chemotherapy \\
\hline 12 & M & 59 & Right & NO & Adenocarcinoma & 6 & Chemotherapy and radiotherapy \\
\hline 13 & M & 38 & Right & NO & Large cell & 6 & \\
\hline 14 & $\mathrm{~F}$ & 40 & Right & NO & Adenocarcinoma & 5 & Chemotherapy \\
\hline 15 & M & 38 & Left & N3 & Adenocarcinoma & 5 & Chemotherapy \\
\hline 16 & $\mathrm{~F}$ & 37 & Left & N1 & Adenocarcinoma & 5 & \\
\hline 17 & M & 53 & Right & NO & Squamous cell & 5 & \\
\hline
\end{tabular}

cally through the epineurium of the cervical and thoracic spinal nerves along the intervertebral foramina. For all but 1 of our patients, multilevel hemivertebrectomy was sufficient to obtain en bloc resection in tumor-free margins and good local control of the disease. In contrast to Grunenwald and colleagues ${ }^{17}$ and Gandhi and coworkers, ${ }^{16}$ we used titanium instrumentation to ensure spinal fixation, and consequently we were able to obtain good quality postoperative MRI scans of the resection areas.

Our results show that this complicated procedure can be performed without operative deaths. However, morbidity was high, with complications occurring in 9 of the 17 cases. In addition, the long operative times, major blood loss, and long hospital stay make the procedure cumbersome. Our results for these parameters differ noticeably from those reported by Gandhi and coworkers, ${ }^{16}$ perhaps because resection was more extensive in our study. All our patients had at least a 3-level hemivertebrectomy with spinal fixation, whereas among the 17 patients operated on by Gandhi and coworkers ${ }^{16}$ only 7 had total vertebrectomy requiring spinal fixation; the 10 other patients had partial vertebral body or transverse process resection without spinal fixation. We consider that these last 2 resections do not aggravate the prognosis of the TI tumor resection, because they do not result in spinal instability. These resections are performed during the transcervical approach. They can be considered as an extended chest wall resection, so we did not include them in this study.

This aggressive procedure is justified, because incomplete resection is associated with reduced survival. ${ }^{14}$ How- ever, patient selection is clearly of the utmost importance. The high postoperative morbidity rate in our study indicates that the procedure should be reserved for young patients free of severe cardiac or respiratory disorders. We considered that massive cortical bone involvement contraindicated the procedure, because this feature is associated with an unfavorable cost/benefit ratio and because frozen sections of bone are difficult to obtain. Nevertheless, such patients must be reevaluated by MRI after induction chemotherapy. If the response to chemotherapy is good, surgical resection can be considered. When there are no contraindications, our technique allows en bloc tumor resection with good locoregional control. The low local recurrence rate $(n=3 / 17)$ is probably attributable to the absence of tumor opening and to the resection in tumor-free margins.

Perioperative radiation has been shown to improve local control. ${ }^{18,19}$ We believe that preoperative radiation therapy is unnecessary, because it fails to affect survival, adds little to the cure rate, ${ }^{3}$ and may induce local tissue fragility, a feature seen in 1 of our patients referred to us after induction radiation therapy. We agree with DeMeester and colleagues $^{3}$ that the tumor should be removed before radiation therapy. For the same reasons, we recommend preoperative chemotherapy only for patients with bulky tumors or with bone invasion. Resection would then be performed only if the response to chemotherapy has been good. Postoperative chemotherapy was given to patients with positive (cervical or mediastinal) lymph nodes. The low local recurrence rate in our study suggests that our position regarding radiation therapy is appropriate. Most of our patients died of distant 


\begin{tabular}{|c|c|c|c|c|c|}
\hline $\begin{array}{l}\text { Vertebral } \\
\text { resection }\end{array}$ & Involved vessels & Involved nerves & Recurrence & Status & $\begin{array}{l}\text { Follow-up } \\
\text { (mo) }\end{array}$ \\
\hline T1-T3 & Subclavian artery and vein & T1 and phrenic nerve & Systemic & Dead & 44 \\
\hline T1-T3 & & T1 and phrenic nerve & Local and systemic & Dead & 20 \\
\hline T1-T3 & Subclavian artery & $\mathrm{T} 1$ & Local and systemic & Dead & 27 \\
\hline $\mathrm{T} 1-\mathrm{T} 3$ & Subclavian artery and vein & $\mathrm{T} 1$ & Local and systemic & Dead & 27 \\
\hline $\mathrm{T} 1-\mathrm{T} 3$ & & $\mathrm{~T} 1$ & & Alive & 70 \\
\hline $\mathrm{T} 1-\mathrm{T} 3$ & & T1 and phrenic nerve & Systemic & Dead & 26 \\
\hline $\mathrm{T} 1-\mathrm{T} 3$ & & T1 and phrenic nerve & Systemic & Dead & 13 \\
\hline $\mathrm{T} 1-\mathrm{T} 3$ & & $\mathrm{~T} 1$ and phrenic nerve & Systemic & Dead & 56 \\
\hline $\mathrm{T} 1-\mathrm{T} 4$ & & $\mathrm{~T} 1$ and phrenic nerve & & Alive & 60 \\
\hline T1-T3 & Subclavian artery and vein & $\mathrm{T} 1$ and phrenic nerve & Systemic & Dead & 16 \\
\hline $\mathrm{T} 1-\mathrm{T} 3$ & & T1 & Systemic & Dead & 9 \\
\hline $\mathrm{T} 1-\mathrm{T} 4$ & & T1 & Systemic & Alive & 28 \\
\hline T1-T3 & $\begin{array}{c}\text { Subclavian artery and } \\
\text { vertebral artery }\end{array}$ & $\mathrm{T} 1$ & Systemic & Dead & 6 \\
\hline $\mathrm{T} 1-\mathrm{T} 4$ & & $\mathrm{~T} 1$ & & Alive & 12 \\
\hline $\mathrm{T} 1-\mathrm{T} 4$ & $\begin{array}{c}\text { Subclavian artery and } \\
\text { vertebral artery }\end{array}$ & T1 & & Alive & 7 \\
\hline $\mathrm{T} 2-\mathrm{T} 4$ & & T1 and phrenic nerve & & Alive & 3 \\
\hline $\mathrm{T} 1-\mathrm{T} 3$ & & $\mathrm{~T} 1$ & & Alive & 2 \\
\hline
\end{tabular}

metastases. Routine postoperative chemotherapy might help to reduce this cause of death.

In conclusion, NSCLCs invading the thoracic inlet and intervertebral foramina that have until now been considered to be inoperable can be completely resected en bloc through a combined anterior transcervical and posterior midline approach. Spinal fixation is the last step of the procedure. However, the long operative time and the substantial blood loss require that this procedure be reserved for carefully selected patients. Perioperative morbidity and mortality are directly related to the experience of the team with spinal, thoracic, and vascular surgery. The number of patients reported on in our study is small, this is only the first study reporting results after vertebral resection for lung cancer in a homogeneous group of patients, with tumor extension and surgical approach similar in all our patients. Our results are preliminary, and studies of larger numbers of patients with longer follow-up times are needed.

\section{References}

1. Dartevelle PG, Chapelier AR, Macchiarini P, Lenot B, Cerrina J, Ladurie FL, et al. Anterior transcervical-thoracic approach for radical resection of lung tumors invading the thoracic inlet. $J$ Thorac Cardiovasc Surg. 1993;105:1025-34.

2. Fadel E, Chapelier A, Bacha E, Leroy-Ladurie F, Cerrina J, Macchiarini $\mathrm{P}$, et al. Subclavian artery resection and reconstruction for thoracic inlet cancers. J Vasc Surg. 1999;29:581-8.

3. DeMeester TR, Albertucci M, Dawson PJ, Montner SM. Management of tumor adherent to the vertebral column. J Thorac Cardiovasc Surg. 1989;97:373-8.

4. Winter RB, Lonstein JE, Denis F, Leonard AS, Garamella JJ. Paraplegia resulting from vessel ligation. Spine. 1996;21:1232-3.
5. Macchiarini P, Dartevelle P, Chapelier A, Lenot B, Cerrina J, Ladurie FL, et al. Technique for resecting primary and metastatic nonbronchogenic tumors of the thoracic outlet. Ann Thorac Surg. 1993;55: 611-8

6. Lapresle P, Missenard G. Hydroxylapatite-coated Diapason screws: first clinical report. J Spinal Disord. 1995;8 Suppl 1:S31-9.

7. Missenard G, Lapresle P, Cote D. Local control after surgical treatment of spinal metastatic disease. Eur Spine J. 1996;5:45-50.

8. Kaplan EL, Meier P. Nonparametric estimation from incomplete observations. J Am Stat Assoc. 1958;53:457-81.

9. Paulson DL. Carcinomas in the superior pulmonary sulcus. $J$ Thorac Cardiovasc Surg. 1975;70:1095-104.

10. Wright CD, Moncure AC, Shepard JA, Wilkins EW, Mathisen DJ, Grillo HC. Superior sulcus lung tumors: results of combined treatment (irradiation and radical resection). J Thorac Cardiovasc Surg. 1987; 94:69-74.

11. Devine JW, Mendenhall WM, Million RR, Carmichael MJ. Carcinoma of the superior pulmonary sulcus treated with surgery and/or radiation therapy. Cancer. 1986;57:941-3.

12. Sartori F, Rea F, Calabro F, Mazzucco C, Bortolotti L, Tomio L. Carcinoma of the superior pulmonary sulcus: results of irradiation and radical resection. J Thorac Cardiovasc Surg. 1992;104:679-83.

13. Maggi G, Casadio C, Pischedda F, Giobbe R, Cianci R, Ruffini E, et al. Combined radiosurgical treatment of Pancoast tumor. Ann Thorac Surg. 1994;57:198-202.

14. Ginsberg RJ, Martini N, Zaman M, Armstrong JG, Bains MS, Burt $\mathrm{ME}$, et al. Influence of surgical resection and brachytherapy in the management of superior sulcus tumor. Ann Thorac Surg. 1994;57: $1440-5$.

15. Muscolino G, Valente M, Andreani S. Pancoast tumors: clinical assessment and long term results of combined radiosurgical treatment. Thorax. 1997:52:284-6.

16. Gandhi S, Walsh GL, Komaki R, Gokaslan ZL, Nesbitt JC, Putnam $\mathrm{JB} \mathrm{Jr}$, et al. A multidisciplinary surgical approach to superior sulcus tumors with vertebral invasion. Ann Thorac Surg. 1999;68: 1778-85.

17. Grunenwald D, Mazel C, Girard P, Berthiot G, Dromer C, Baldeyrou $\mathrm{P}$. Total vertebrectomy for en bloc resection of lung cancer invading the spine. Ann Thorac Surg. 1996;61:723-6. 
18. Shaw RR, Paulson DL, Kee JL Jr. Treatment of the superior sulcus tumor by irradiation followed by resection. Ann Surg. 1961;7:2940.

19. Van Houtte P, Mac Lennan I, Poulter C, Rubin P. External radiation in the management of superior sulcus tumor. Cancer. 1984;54:223-7.

\section{Discussion}

Dr Douglas E. Wood (Seattle, Wash). I congratulate Fadel and colleagues on this thoughtful analysis of their group's experience with this subset of patients with locally advanced NSCLC. The Marie-Lannelongue group has consistently led the development of extended surgical resections for T4 tumors that have generally been considered unresectable. They have reported their large experience with carinal resection, superior vena cava resection, and more recently the cervical thoracic approach for anterior Pancoast tumors.

In this report they took resection of Pancoast tumors to yet another level, developing an approach that allows a complete en bloc surgical resection of tumors involving the intervertebral foramina. T4 tumors were originally defined by perceived anatomic limitations to resection, not because of an inherent biologic behavior that precluded surgical benefit. As surgeons develop successful techniques for achieving a complete resection, subsets of these locally advanced primary tumors can be treated surgically with curative intent. Last year the thoracic group from Massachusetts General Hospital reported their outstanding results in patients undergoing carinal pneumonectomy, and now Fadel and colleagues challenge us again with a group of patients who would previously have been referred to radiation oncology for definitive therapy.

It is important to emphasize the selective nature of these reports of aggressive surgery for T4 tumors. As surgeons we congratulate ourselves on modest survivals among patients with a very poor prognosis. However, during our evaluations we exclude the vast majority of patients with T4 disease: those with bulkier, more advanced disease; older patients; patients with significant comorbidities; and those with poor performance status. We refer these patients to our medical and radiation oncology colleagues, and these patients do have the prognosis that we expect from stage IIIb lung cancer. We select perhaps $1 \%$ or $2 \%$ of patients with $\mathrm{T} 4$ disease, those who are young with good performance status and with limited T4 invasion that allows surgical resection. I am an advocate for this aggressive approach, but I think that it is important for us to be honest in admitting that the modest patient survival may be due as much to a more favorable selection bias as to our surgical prowess. It is humbling to see that Fadel and colleagues had a 5-year survival of only $20 \%$ and a median survival time of only 27 months. Indeed, this is better than for most patients with stage IIIb disease, but at a significant cost of surgical morbidity and average 30-day hospital stay. It is possible that similar median survival statistics could be obtained with definitive chemoradiotherapy in an outpatient setting. This is a legitimate possibility, given that we now have experience in induction chemoradiotherapy for stage IIIa lung cancer that achieves a complete response in $20 \%$ to $30 \%$ of cases.

Dr Fadel, do you have information on the outcomes of similar patients in your institution who underwent chemoradiotherapy instead of surgical resection?
Dr Fadel. More than two thirds of these patients were not operated on because of general condition: severe cardiac condition, respiratory disease, or tumor extension. Those patients were referred to oncologists, and most of them died within a year. None were alive 3 years later. It has already been proved that only surgical resection of these tumors can provide long-term survival.

Dr Wood. You have taken great pains in your preoperative staging of the primary tumor to assure yourself of the ability to achieve a complete resection, and in this you have been successful. It is clear that N2 and N3 nodal disease is a marker of systemic disease, with virtually all patients dying of systemic disease. Previous reports of surgery for $\mathrm{T} 3$ disease have shown no survival among patients with T3 N2 disease, and most would consider N2 disease a contraindication to an extended surgical resection. What techniques do you use to evaluate the mediastinal lymph nodes? Do you perform a positron-emission tomographic scan or mediastinoscopy? If not, why does it appear that you are less stringent with the criterion of nodal involvement than you are with those of a primary tumor and the patient's age and performance status?

Dr Fadel. This is really an important question. We performed mediastinoscopy when the preoperative CT scan demonstrated large mediastinal lymph nodes. Since last year, however, we have been performing routine positron-emission tomography. When the tumor staging is $\mathrm{N} 2$, we do not operate. The patients in this series with $\mathrm{N} 2$ disease had it discovered during the mediastinal dissection. Now if we see subcarinal nodes larger than $1 \mathrm{~cm}$ on CT scan, we perform preoperative chemotherapy.

Dr Wood. Then, how many patients did you exclude because of N2 or N3 nodal disease?

Dr Fadel. I do not remember the exact number, but maybe around 20, about the same as we included.

Dr Wood. Of the patients who have been followed up for longer than 1 year, all but 1 have died of systemic recurrence, further evidence that systemic disease remains the problem limiting survival among these patients with locally advanced tumors. Do you now recommend chemotherapy for all patients before resection, or do you still treat patients predominantly surgically and with adjuvant radiation?

Dr Fadel. I do not think that routine preoperative chemotherapy is recommended, because it does not effect a cure of the tumor. We recommend chemotherapy for bulky tumors and when there is vertebral body invasion detected by MRI. It might be interesting to do routine chemotherapy after the operation, because most of these patients die of distant recurrence.

Dr Wood. I guess my point is that you are using therapy for local control, but your problem is systemic disease. You only had 2 survivors, 2 long-term disease-free survivors, and everyone else died of systemic disease, so you might rethink your chemotherapeutic regimen. Thank you for sharing your experience and continuing to advance the ability to offer some additional patients with lung cancer the hope of a prolonged disease-free survival.

Dr Richard Whyte (Stanford, Calif). I think that Dr Wood was right on the mark here when he talked about the distant failure rate. My question, however, is about the local failure rate. There were, I think, 3 patients who had local failures. My question is, how do you ensure that these margins are negative, particularly with respect to a bony margin? 
Dr Fadel. These patients died of metastasis. We routinely performed postoperative MRI every 6 months to detect local recurrence. Most of these patients die of cerebral or hepatic metastasis.

Dr Whyte. How do you ensure that your margins are negative?
Dr Fadel. It is not possible to do frozen-section analysis on a vertebral body. So we have only postoperative confirmation of vertebral invasion. Therefore we do not operate on patients with vertebral body invasion evident on MRI, because we are not sure that we can do en bloc resection and have tumor-free margins.

Online-www.aats.org Now you can get The Journal of Thoracic and Cardiovascular Surgery online. The Journal online brings you faster delivery time, easy searching of current and back issues, links to PubMed, AATS, WTSA, and other important sites, and more. Visit the Journal online today.

\section{Receive tables of contents by e-mail}

To receive the tables of contents by e-mail, sign up through our Web site at http://www.mosby.com/jtcvs

Choose E-mail Notification

Simply type your e-mail address in the box and click the Subscribe button. Alternatively, you may send an e-mail message to majordomo@mosby.com. Leave the subject line blank and type the following as the body of your message: subscribe jtcvs_toc

You will receive an e-mail to confirm that you have been added to the mailing list.

Note that TOC e-mails will be sent out when a new issue is posted to the Web site. 